\title{
PAISAGENS RURAIS CULTURAIS: OS NOVOS ESPAÇOS DA RESILIÊNCIA
}

\section{CULTURAL RURAL LANDSCAPES: NEW RESILIENCE SPACES}

\author{
Luisa Spagnoli \\ Research fellow and lecturer in geography \\ Instituto de História da Europa Mediterrânea, \\ Conselho Nacional de Pesquisa \\ luisa.spagnoli@uniroma2.it
}

\begin{abstract}
RESUMO
Vivemos emuma época em que a paisagem está em todos os lugares, proposta à atenção de todos, à participação coletiva, no centro de um intenso debate disciplinar e político, em virtude do qual confrontamo-nos seja em âmbito teórico que operativo. Em particular, a partir da bem conhecida redescoberta paisagística, perfilada nas últimas décadas do século passado, colocando em evidência a valia polissêmica da categoria conceitual da paisagem, cuja "ambiguidade" semântica é reconduzível essencialmente à sua condição objetiva e subjetiva. Portanto, os novos endereços de pesquisa, assim como as novas "tendências" projetuais, mostram uma crescente sensibilidade no que diz respeito à paisagem, com a finalidade de definir programas de intervenção no território sempre mais orientados à consideração e valorização "holística" dos mesmos. Neste contexto, esta contribuição entende realçar a categoria "cultural" das paisagens rurais e individualizar medidas sustentáveis voltadas à reconfiguração das relações entre espaços urbanos e rurais. O objetivo consiste em propor atenção precípua nas paisagens agrárias, colocando em evidência as políticas e as "opções" projetuais endereçadas a estimular novas formas de desenvolvimento rural, a partir das quais propor uma regeneração dos espaços da agricultura, privilegiando $o$ paradigma da multifuncionalidade e da integração.
\end{abstract}

Palavras Chave: paisagem rural, agricultura, políticas agrícolas e dedesenvolvimento rural

\footnotetext{
ABSTRACT

At the present time, landscape is everywhere. It is always there, as the focus of everyone's attention and participation, and as the center of a strong disciplinary and political debate, on a theoretical and operational level. In particular, from the wellknown landscape revalorization, which has been developing throughout the last decades of XX century, we saw the increasing importance of the polysemous value of landscape as conceptual category, which semantic "ambiguity" is connected to its objective and subjective condition. The new aims of research, as well as the new planning "tendencies", show an increasing sensitivity about landscape, with the final purpose of defining the intervention programs on territories, through an "holistic" consideration and valorization. With this in mind, this contribution wants to highlight the "cultural" 
category of rural landscapes, and to discover sustainable measures aimed to reconfiguring the relation between urban and rural spaces. The main intent is proposing a greater attention on the rural landscape, pointing out the policies and planning "options" aimed to stimulate new forms of rural development. From there, we can propose a regeneration of agricultural spaces, encouraging a paradigm of multifunctionality and integration.

Keywords: rural landscape, agriculture, agricultural policies, rural development policies

\section{Premissa. A paisagem a caminho da aquisição da dimensão cultural}

$\mathrm{O}$ crescente interesse pela paisagem, como se sabe, amadureceu a partir das últimas décadas do século passado, solicitando seja a reelaboração do aparato teóricometodológico que aos poucos foi se fragmentando e disperdendo em um corpus não orgânico de interpretações e leituras heterogêneas, seja a definição das ações que em âmbito local e supralocal impulsionaram maior responsabilidade e uma consciente programação territorial.

Em outras palavras, deparamo-nos com um conceito que se enriqueceu em virtude especialmente do confronto que amadureceu no seio dos diversos ambientes disciplinares, cujas considerações científicas se "carregaram" ao propor uma sistematização definitiva do paradigma paisagístico. Resultando em um quadro poliédrico, que nada mais é que o reflexo da complexidade paisagística.

Todavia, este leque de leituras e nuances possíveis, multiplicidade de discursos sobre os quais se verificouou, conduz a duas tendências fundamentais: a paisagem como lugar de sinais a serem decifrados e a paisagem como espaço de concretização objetiva, que por sua vez reenviam à necessidade de referir-se às ligações entre os processos materiais e os processos de representação; à aquisição de uma tendência de materialização dos sinais, em virtude do «giococombinatodifattoriecologici e umani»*que nesta se manifestam, e à assunção de formas intangíveis, que refletem da paisagem o «manto divaloriattribuiti ad undeterminato contesto territorialeche assurge essenzialmente a spazioculturale» (Vallega, 2007, pp. 49-52).

E éespecialmente no âmbito geográfico, talvez em consideração à abordagem maioritariamente holística da disciplina e a sua tendência a agrupar as dimensões cultural, social, econômica e política, que ganharam forma diversos pontos de vista. $\mathrm{Na}$ verdade, foi com a Geografia que aconteceu a transformação definitiva do conceito de 
paisagem: de fenômeno estético a objeto de pesquisa (1). Da acepção de paisagem «come sintesiastrattadiquellivisibili» (Biasutti, 1947, p. 3), chegou-se à conclusão do seu ser «una costruzione» até mesmo «mentale interna concuinoirappresentiamoil mondo esterno» (Dematteis,1989, p. 451); quer dizer, um «fenomenodisignificazione e dunque cultura» (ibidem).

É em tal polissemia que está inserida a sua mais-valia cultural, da qual certamente hoje em dia tem-se maior consciência, também no âmbito da planificação, cuja ação «deve partirepropriodalriconoscimento dei significati e dei valori localmente attribuiti ai caratteridiunterritorio» (Zerbi, 1993, p. 83). Assim, na ambivalência semântica da paisagem criam-se também pressupostos razoáveis para a definição de uma modalidade projetual projetada para uma dinâmica inovadora. Portanto, a sua ambiguidade dual não tem importância apenas no nível da reflexão teórica, mas também em termos de praxe territorial: a objetividade sem a subjetividade, e vice-versa, não produz nenhum resultado. O projeto da paisagem encontra a sua razão de ser ao saber mediar ambas as estâncias.

Chegar a uma projetualidade que restitua sentido à paisagem, empregar - como escreve Roberto Gambino (1997, pp. 29-30) -a produção social do território, significa contemplar uma leitura objetiva dos elementos paisagísticos, assim como a «soggettività dei processidiproduzionedelpaesaggiostesso», na medida em que é um dado de fato que esta seja um mundo constituído de elementos e, ao mesmo tempo, de indivíduos que olham, percebem e sentem, restituindo-nos a plenitude dos lugares, dos seus significados e valores culturais. Sob estes pressupostos, a paisagem mostra-se como uma realidade pronta para sustentar «un'azionediriforma» (ibidem), que se alimenta e redefine na dimensão material do território e na pluralidade de sujeitos que a habitam e a produzem.

A tal necessidade, de releitura complexa das paisagens, fazem referimento sempre mais experiências que, partindo de novos modelos de desenvolvimento, são capazes de fundar «lapropriasostenibilità e durevolezza» - diria Alberto Magnaghi (2011, p. 112)sobre a «valorizzazionedellepeculiaritàpatrimonialilocali», expressando-se através de uma renovada demanda social da paisagem, orientada a restituir principalmente valor central às áreas rurais residuais e àquelas áreas em que o urbano e o rural confudem-se, 
contudo não se permeiam favoravelmente. Ou melhor, perdem a especificidade de um e do outro contexto, terminando por gerar um amálgama indistinto.

Em função disso, caminha-se progressivamente na direção de uma definição e da adoção de políticas capazes de propor estratégias de requalificação dos assim chamados territórios "frágeis", diretas a estimular a pluralidade das funções sociais, culturais, ecológicas, às quais recorrer para ativar a prática da agricultura multifuncional e da proximidade, da cadeia curta, da valorização das biodiversidades e das produções locais. O conjunto de tais experiências de caráter multifuncional conduz, em outras palavras, a uma refuncionalização das áreas agrícolas urbanas e rurais.

Esta é a chave de leitura para propor uma reflexão sobre a categoria cultural das paisagens rurais e para individualizar medidas sustentáveis orientadas à reconfiguração das relações entre espaços urbanos e rurais. $\mathrm{O}$ objetivo consiste em propor uma atenção precípua à paisagem agrária, colocando em evidência as políticas e as "opções" projetuais endereçadas a estimular novas formas de desenvolvimento rural.

Atribuir nova territorialidade à paisagem é uma estrada que pode ser percorrida, sob a ótica de uma estratégia de governo territorial multifuncional e integrada, centrada na valorização do papel da agricultura e dos atores envolvidos no processo produtivo. De fronte à perda de espaços, a agricultura renova-se, operando em termos de reconquista cultural e social dos mesmos.

\section{As paisagens agrárias e a"extraordinalidade" dos locais da Unesco}

Se, como testemunham as páginas anteriores, a consciência da dimensão cultural da paisagem é hoje em dia amplamente difundida e verificada, especialmente no âmbito do debate científico, não é igualmente "praticada" e, portanto, plenamente operativa no plano das políticas de gestão da paisagem, muito menos na agrária.

Persistem dificuldades no reconhecimento do valor sistêmico desta última, do significado de suas características e das suas peculiaridades histórico-culturais, chamando a atenção prioritariamente sobre a sua mais-valia econômica e produtiva. Em uma palavra, continua, às vezes, a prevalecer a ideia de sua coincidência com o uso do solo.

Mas, a paisagem agrária é bem diferente. Segundo a leitura de Emilio Sereni, podese entendê-la como «quella forma che l'uomo, nel corso e ai fini delle sue attività 
produttive agricole, coscientemente e sistematicamente imprime al paesaggio naturale» (Sereni, 2010, p. 29). Trata-se de uma construção complexa, estruturada no tempo, que expressa a cultura da comunidade que superintendeu a sua concepção, dando vida a um potente artefato cultural, o qual por sua vez expressa «un fare, unfarsidi [...] genti vive» (ibidem, p. 19).Portanto, de fato, paisagens culturais, nas quais se reflete a sabedoria territorial «diquellegenti vive» que souberam desdobrar as suas atividades produtivas e as suas "intenções" sobre o território, ativando contínuas e sistemáticas transformações que, progressivamente, fizeram da paisagem fruto de uma ação humana e da percepção social.

Progressos, endereçados a acolher a dimensão cultural das paisagens rurais, estão sendo realizados na Itália e na Europa também com a adoção de catálogos, instrumentos de conhecimento em razão dos quais solicitar propostas projetuais para tutelar e valorizar dinamicamente as realidades paisagísticas. Ainda que em alguns casos se trate de experiências reconduzíveis em parte à classificação - como, a título de exemplo, os projetos The Eucalandproject: EuropeanCultureexpressed in AgriculturalLandscapes e o Nazionale dei Paesaggiruralistorici(2) - também é verdade, por outro lado, que ainda antes de projetar o território e os espaços agrícolas, seja indispensável colocar em prática uma atividade cognitiva com base na qual proceder a individualização dos bens paisagísticos e das suas especificidades culturais. Neste sentido, o Código dos Bens Culturais (Decreto Lei de 26 de março de 2008, $\S 1^{\circ}$, art. 3) confirma que apenas «una adeguata attività conoscitiva può indirizzare verso una tutela, in virtù della quale individuare il patrimonio culturale e garantirne la protezione e la conservazione per fini di pubblica fruizione». Daí a importância de uma avaliação inicial, com recurso, se necessário, ao instrumento da catalogação, para focalizar os diversos fenômenos que caracterizam as paisagens rurais. Com o Catalogo Nazionale, tentou-se a estrada da compreensão dos processos que ao longo do tempo concorreram para a atual definição das paisagens italianas.

Partindo do pressuposto que estas últimas correspondem a «sistemi complessi basati su tecniche ingegnose e diversificate che hanno fornito un contributo fondamentale alla costruzione e al mantenimento del nostro patrimonio storico, culturale, naturale [...]» (Agnoletti, 2011, p. 5), o objetivo consiste na aplicação de critérios da significação, integração, vulnerabilidade (3), orientados para a descrição de características de 
permanência.Estes os pressupostos para definir uma projetualidade que, alavancada na importância do reconhecimento das sedimentações do passado, adquira a consciência da necessidade de projetar-se no presente e no futuro e, assim fazendo, lance as bases para a renovação do patrimônio territorial local. Portanto, a insistência sobre a dimensão cultural das paisagens agrárias e, consequentemente, sobre a imanência histórica dos processos transformativos, conduz à redescoberta de um conjunto de práticas, tradições e valores que participam da inteira existência do homem. E é a partir destes elementos que se pode prospectar um projeto futuro, dinâmico, de interpretação e de recuperação do território.

Contudo, é aceitável a objeção que o êxito de tal "prática" poderia conduzir à realização de um elenco classificatório unicamente de tipo tradicional, centrado na individualização de meras unidades paisagísticas, recolhidas principalmente com base nas características físicas da paisagem. No caso do Catalogo Nazionaledei paesaggiruralistorici, há mais a intenção de privilegiar a pesquisa destinada às peculiaridades histórico-culturais da mesma, na tentativa de chegar ao coração das estruturas culturais e, assim, acolher a já evocada concepção sereniana de que as paisagens são o resultado das formas impressas pelo homem, com a consciência e a sistematização, sobre a natureza.

Apesar disso, não se pode não reconhecer a experiência da catalogação, dirigindo-se a poucas e excepcionais paisagens, tais como os "Campi baulatidelCasalasco" na Lombardia, os "CastagnetidelmedioLavino" na Emília-Romanha ou ainda os "CastagnetidelReventino" na Calábria, os "VignetiterrazzatidellaValtellina" naLombardia, assim como os "Santa Maddalena" no Alto Ádigee os "VignetidiLamole" na Toscana etc., limites da ação da valorização apenas aos casos de extraordinariedade.

Em linha com o que foi recém descrito, ainda que com uma visão mais especificamente sistêmica e certamente antecipatória, coloca-se a Convenzione UNESCO per laProtezionedelPatrimonioMondialeCulturale e Naturale, que, entre outros, compartilha um dos princípios propostos no Catalogo, o da integridade das paisagens, como pressuposto fundamental para descrever a permanência das suas características.

A partir de tal instrumento normativo, renovado no início dos anos noventa do século $\mathrm{XX}$, desenvolveu-se o interesse em relação às paisagens agrárias e rurais, consideradas 
uma categoria específica de "paisagens culturais" a proteger e a valorizar. Adotada durante a XVII Sessão da Conferência geral da Organização das Nações Unidas para a educação, a ciência e a cultura (UNESCO), em 16 de novembro de 1972, a Convenzione definiu o patrimônio cultural e natural e as modalidades de operar sustentavelmente no território, adotando uma política de cooperação entre os Estados (art. 6, parágrafos 1 e 2).

O Documento atua uma distinção entre patrimônio cultural e natural, cuja proteção não se estende explicitamente à categoria da paisagem. Todavia, a seguir à revisão das OperationalGuidelines, em razão das recomendações elaboradas no âmbito da reunião internacional realizada em Le Petite Pierre, na França, em 1992, vinte anos depois da adoção da Convenzione, a UNESCO trabalhou para estender o conceito da categoria dos "locais", compreendida na "herança cultural", incluindo a paisagem como «opera dell'uomo o opera congiuntadell'uomo e della natura» (art. 1, Convention, 1972). E, consequentemente, sendo consideradas «"le opere umane, o combinazioni di opere umane" come parti delle eredità culturali, l'oggetto dell'intervento [è stato] configurato come "paesaggio culturale". Da quel momento l'iscrizione dei siti nell'ambito della Lista [dei beni] del Patrimonio Mondiale [...], ha compreso anche i paesaggi culturali»» (Vallega, 2008, pp. 26-27) (4). Entre estas devem ser tuteladas aquelas consideradas mais representativas dos diferentes países do mundo: criações «complesse frutto del rapporto costruttivo tra uomo e natura, [che] esprime [...] la lunga e intima relazione tra i popoli e il loro ambiente naturale» (Petrillo, Di Bella, Di Palo, 2012, p. 217).

Portanto, no sentido de obras resultantes da ação humana que se associaram à influência da natureza, mostrando diferentes vocações e ilustrando a evolução da estratificação das manifestações paisagísticas (5): as paisagens rurais e as agrosilvopastoris participando do amplo quadro conceitual de tais categorias. Certamente, trata-se no entanto e sempre de paisagens que mostram traços de significatividade e excelência em virtude dos valores dos quais são revestidas (6).

\section{A agricultura e a valorização das paisagens rurais}


A ação da tutela que a Convenzione UNESCO exerce, destina-se às paisagens culturais, também com vocação agrícola, mas que, todavia, apresentam características de excepcionalidade tais ao ponto de fazer delas merecedoras da inscrição na Lista, negligenciando assim numerosas outras realidades que respondem aos ritmos da cotidianidade e apresentam caraterísticas fragmentárias e de marginalidade, mas que refletem o mundo vivido pelo homem, excepcional ou degradado, "frágil" ou "forte".

Foram principalmente as paisagens agrícolas tradicionais que perderam grande parte das suas atrações, em nome da imposição de uma lógica urbanocêntrica em obediência a um quadro eminentemente funcional. Mas não foi apenas isso. Progressivamente verifica-se a tendência à difusão no território nacional, como também em escala europeia, de uma malha urbana alargada, de um espaço indistinto, dificilmente conotativo como urbano ou rural. Tratam-se de territórios rurbanos e periurbanos, «in cui convivono stabilmente caratteri e segni differenti, derivanti da matrici e dinamiche in alcuni casi contrapposte, quali quelle tipiche dei processi di urbanizzazione e, al loro opposto, quelle di ruralizzazione» (di Mario e Pascale, p. 27).

É um dado de fato que se na Europa as áreas agrícolas perdem progressivamente terrenos, na Itália, todavia, a superfície agrícola utilizada (SUA) parece reduzir-se em proporção menor em relação ao passado: dos $-12 \%$ do período intercensitário precedente a $-2,3 \%$ de 200-2010. Tal tendência pode ser explicada, talvez, com uma atenção pouco a pouco maior ao cuidado dos lugares e provavelmente com o progressivo empenho por parte dos programas de desenvolvimento rural regionais, que, se por um lado estão ainda em fase de "experimentação", por outro, revelam-se muito sensíveis ao ativar iniciativas sustentáveis e finalizadas a valorizar as vocações territoriais específicas. Não obstante - como indicado no Rapporto da SocietàGeografica Italiana - o consumo do solo e da cimentação dos espaços destinados à agricultura são fenômenos em expansão, principalmente se referidos à porção setentrional do País que detém a primazia das superfícies artificiais (Pollice, 2012, p. 57) (7). Considere-se também a circunstância que ao alimentar a "artificialização" dos terrenos agrícolas contribui-se para a «diffusionenelleareeperiurbane e ruralidigrandistrutturedelterziariocommerciale» (ibidem, p. 57). Em outras palavras, mesmo que o incremento das superfícies urbanizadas mostre uma intensidade pouco a pouco mais reduzida, a agriculutra continua a sofrer um relevante depauperamento das 
áreas férteis (ibidem, pp. 15-16)(8).

No entanto, mesmo se a situação não é das mais confortáveis, não se pode ignorar que o processo de renovação da Política Agrícola Comunitária (PAC), já há algumas décadas endereçado à redescoberta dos valores territoriais e à requalificação das culturas tradicionais locais, tentou contribuir para melhorar o quadro evolutivo da agricultura. No período entre os anos oitenta e os anos noventa do século XX, na verdade, ativa-se um processo que leva à maturação da consciência da falência das medidas até aquele momento adotadas, na tentativa de proteger as áreas agrícolas e os recursos naturais. Sobre tais pressupostos, a PAC, de uma abordagem setorial, passou a um modelo de desenvolvimento baseado na multifuncionalidade e na integração. A nova meta consiste em abrir ao mundo agrícola novos cenários onde «riscoprireritmi e valori sui qualiècresciuto» (ibidem, p. 50) (9).

A paisagem rural, assim como os espaços marginais da agricultura, encontra razão de ser no quadro revisado da PAC e das mais recentes políticas territoriais, na origem da refuncionalização dos espaços agrícolas, seja dos que perderam a sua própria destinação de uso, seja daqueles que sobreviveram de forma residual.

A renovação da agricultura, a introdução de bestpracticesecossustentáveis, a releitura da relação cidade-campo, a aplicação do modelo de desenvolvimento local, baseado na integração entre os elementos paisagísticos, contribuem para regenerar as paisagens agrárias, para fecundar novas ideias para novos "usos" e projetualidades. Torna-se central o reconhecimento da "espessura" cultural das paisagens rurais e dos próprios valores, que servem como base para um desenvolvimento centrado no reequilíbrio ambiental e territorial. Parte-se de uma agricultura "regenerada", atenta às características locais e qualitativas dos territórios, para se chegar à construção das paisagens, cada vez mais desvinculadas a lógicas, medidas e estratégias de intervenção “convencional", capazes de empreender o caminho da nova territorialidade, que reflete, por sua vez, o percurso direto à sustentabilidade e à valorização do conjunto de seus elementos - culturais e ecológico-ambientais; materiais e imateriais - com a finalidade última de criar uma pluralidade de serviços para oferecer à coletividade.

\section{O valor cultural das paisagens agrárias italianas nas políticas de desenvolvimento}


rural.

Uma conclusão

Das considerações até aqui delineadas, emerge a necessidade de "sustentar" as paisagens agrícolas seja rurais que periurbanas desejando uma solução inovadora para a agricultura: trata-se de percorrer não tanto a estrada da "rentabilidade", mas principalmente a opção da polifuncionalidade, de maneira a solicitar a formação dos contextos territoriais sustentáveis.

Se a mais-valia cultural das paisagens agrárias é um elemento fundamental para as “medidas" UNESCO, não é outrossim explícita no âmbito das políticas de desenvolvimento rural em nosso País. Se amadureceram em escala transdisciplinar, a consciência do papel fundamental que a agricultura está assumindo, a vantagem de uma regeneração das paisagens e das atividades de desenvolvimento local, não são igualmente difusas no seio das políticas do governo territorial. Todavia, no Piano StrategicoNazionale per losvilupporurale(PSN), um documento fundamental ao qual as programações regionais devem recorrer, sublinha-se a necessidade da recuperação e da proteção da paisagem cultural, frente ao incentivo dos processos de degradação e à ineficiência das políticas de desenvolvimento. O ponto de partida é a consciência de que a paisagem italiana é o reflexo territorial de uma história milenar pluriestratificada, que porta consigo a identidade cultural do País. E é, como tal, «una risorsa fondamentale [...], configurandosi come elemento chiave per lo sviluppo turistico e per la biodiversità legata alla qualità degli spazi coltivati [...] e rappresentando un aspetto caratterizzante la qualità della vita nelle aree rurali» (Piano Strategico Nazionale, 2010, p. 26).

Os objetivos do PSN são destinados às áreas rurais do contexto italiano complexamente interessadas, para responder às "exigências" definidas em nível comunitário: «migliorare la competitività del settore agricolo e forestale; valorizzare l'ambiente e lo spazio rurale attraverso la gestione del territorio; migliorare la qualità della vita nelle zone rurali e promuovere la diversificazione delle attività economiche» (ibidem, p. 47). As finalidades em questão, que remetem, por sua vez, aos quatro “eixos" para a programação do desenvolvimento rural 2007-2013 estabelecidas pelo Regulamento (CE) n. 1698/2005, são contempladas não tanto segundo a lógica de cada um dos "eixos", mas integradas nas formas consideradas mais oportunas em nível regional: a valorização das áreas rurais adquire uma valor adicionado à luz da 
integração dos recursos ambientais e culturais das próprias paisagens.

De fato, porém, seja no âmbito das medidas do PSN seja nas políticas agrícolas e do desenvolvimento rural, consideradas no conjunto, definem-se estratégias de desenvolvimento territorial marcadas por um endereço predominantemente de caráter geral.

No entanto, parecer emergir do Regolamento (CE) a oportunidade de levar em consideração uma «diversitàdisituazioni», nas quais podem entrar «le zone ruraliremote colpite da spopolamento e declino e le zone ruraliperiurbanechesubisconola pressione dei centriurbani» (art.11). Mas trata-se do reconhecimento da diversidade dos contextos rurais europeus que, de qualquer maneira, se limita a uma consideração complexa, que restitui uma visão do conjunto, sem adentrar efetivamente na especificidade das características identitárias das paisagens.

A razão da escassez de medidas de intervenção específicas e diferenciadas, todavia, pode ser reconduzida à dificuldade ainda difusa na individualização, plenamente consciente, das peculiares identidades paisagísticas, as quais correspondem criticidade e potencialidade que deveriam ser "interpretadas" de maneira variada. Também em escala regional, os Programas de Desenvolvimento Rural (PSR, na sigla original), ao apresentar medidas de planejamento, tratam a paisagem desarticulada da complexidade de seus fatores econômicos, ambientais, sociais, negligenciando o elemento identitário, necessário para alcançar «unadeguatolivellodicompetitività» (Brunori, Fastelli, Rovai, 2013,p. 148) (10).

Em um olhar complexo, se as políticas agrícolas e de desenvolvimento rural, em escala nacional e supranacional, estão progressivamente emancipando-se de um modelo até agora predominantemente centrado na «omologazionetecnologica e produttiva» (Tempesta, 2011, p. 144), o reconhecimento da dimensão cultural das paisagens agrárias e rurais ainda encontra dificuldades para afirmar-se e difundir-se plenamente, não apenas em nível político-normativo, mas também no plano local. Apesar disso, cresce a consciência de que é próprio das comunidades locais que deve iniciar a recuperação da identidade e da diversidade das paisagens, entendidas como «un elemento diforzadelpropriosviluppo futuro» (ibidem). Para que isso aconteça, não se pode deixar de abraçar a ideia da necessária integração das ações previstas pela planificação territorial e paisagística com as intervenções da política agrícola e de desenvolvimento 
rural.

\section{Notas}

* Respeitando a escolha da autora, as citações foram mantidas na língua original para conservar a rigorosidade científica do artigo. Lembra-se que o substantivopaisagem é masculino em italiano e isto,em algumas passagens do texto, pode provocar uma imprecisão na concordância da frase, sem modificar, porém, o sentido e a compreensão da mesma.

(1)Tal translação teve lugar no seio da cultura geográfica alemã em pleno século XIX. Figura de destaque iniciou o processo de transformação do conceito de paisagem, Alexander Von Humboldt, extraordinário viajante e pioneiro da geografia moderna, soube captar a essência objetiva do Mundo na paisagem, subtraindo-o assim da pura contemplação estética.

(2) O projeto Educaland, concluído em 2009, ativado pela Universityof Cambridge (UK) e apoiado pela Eucaland network, com a contribuição de numerosos parceiros internacionais, servindo-se dos fundos da Comissão Europeia para o programa cultural 2007-2013. A ideia que guiou o projeto consiste na necessidade de valer-se de uma abordagem holística, interdisciplinar e multicultural para avaliar as paisagens rurais europeias. Tratou-se principalmente de descrever as paisagens, colocando em evidência as características fundamentais e os benefícios culturais, sociais e psicológicos que as comunidades possam tirar dessas paisagens, em virtude da riqueza dos valores de que são expressão. A partir destes pressupostos, impostou-se uma classificação das paisagens agrárias europeias, recorrendo a parâmetros qualitativos que privilegiaram a descrição dos contextos indagados, das especificidades e das atividades produtivas, mas, principalmente, a análise das dinâmicas históricas e agrícolas.

(3) A significatividadeé chamada em causa para sublinhar o papel fundamental do conjunto de valores que a paisagem exprime, em particular, refere-se à «"persistenzastorica" dellastrutturadegliordinamenticolturalipresenti» (Agnoletti, 2010, p. 25). As paisagens assim classificadas, baseadas neste critério, são aquilo que poderemos definir "histórico-culturais", cuja «lenta evoluzione mostra una significativa armonia integrativa traaspettiproduttivi, ambientali e culturalidi una data area o regione» (ibidem). A integridade é chamada para descrever o estado das paisagens e, consequentemente, as suas qualidades e potencialidades, também na razão do delineamento das políticas de proteção e valorização. A vulnerabilidade enfoca os fatores de risco que vão ao encontro destas paisagens que precisam de intervenções de requalificação.

(4) Das paisagens culturais, segundo a mais recente acepçãoda UNESCO, entende-se sublinhar a predisposição para ilustrar «l'evoluzione della società umana e dell'insediamento nel corso del tempo, per effetto dell'influenza di condizionamenti fisici e/o di opportunità determinate dal loro ambiente naturale e dall'insorgenza di fattori sociali, economici e culturali, esterni e interni» (Vallega, 2008, p. 27).

(5) A UNESCO subdividiu as paisagens culturais em três categorias. A primeira é representada pelas «paesaggibendefiniti e creatiintenzionalmentedall'uomo», na qual podem ser deixados os jardins e os parques.A segunda constitui-se das «paesaggi evoluti organicamente», em outras palavras: «che rispondono a un originario imperativo di natura sociale, economica, amministrativa, e/o religiosa, e si sono evoluti fino a conseguire le forme attuali». A terceira: as paisagens culturais «associativi»; neste caso o elemento natural reveste-se de valores artísticos, religiosos ou culturais(Vallega, 2008, p. 27).

(6) Antes de obter tal reconhecimento, entre 1992 e 2002, aconteceram numerosos encontros no curso dos quais foi colocada particular atenção nas paisagens agrícolas e, especialmente, nas vitivinícolas. Entre os locais ligados à vitivinocultura, inscritos na lista de patrimônio Mundial da Humanidade: o francês de Saint Emilion, o português da região vinícola do Alto Douro e a região do vinho Tokaj na Hungria, que, mesmo se cada um foi selecionado pelas próprias especificidades, testemunham o alto valor paisagístico das regiões consideradas únicas. Representam o fruto da sabedoria territorial adquirida, de uma consciência e de uma experimentação, que refletem a incessante e contínua intervenção por parte do homem, cuja ação integrou-se muito bem à natureza, desenhando uma condição sistêmica coevolutiva. E assim também a inscrição da paisagem cultural italiana da costa da Liguria"Portovenere, Cinque Terre e as ilhas de Tino, Tinetto e Palmaria,", que, não obstante não tenha sido proposta por motivos propriamente vitivinícolos, é considerada pela UNESCO uma paisagem de intenso valor cultural, por concentrar em si a história do modelamento humano que durou séculos, e no qual existe uma tradição particularmente ligada às culturas das parreiras e das oliveiras.

(7) Na Itália, a superfície artificial é atestada em torno a um valor de 7,3\% (Pollice, 2012, pp. 56-57).

(8) Deve-se dizer, porém, que embora as empresas dedicadas à agricultura tenham sofrido «una 
flessionedialmenounterzo», a situação não seja generalizada, porque na Itália, de acordo com os contextos regionais, as variações são mais ou menos intensas. Tais diversificações territoriais refletem-se também no âmbito das "tipologias" de agriculturas práticas: pode-se estar em presença de uma agricultura que ainda sofre a marginalização de algumas realidades territoriais em que é desenvolvida; ou então de uma agricutltura "post-moderna" que introjeta as estâncias inclusive participativas e, principalmente, de integração setorial (ibidem, p. 17).

(9) A nova reforma da PAC (2014-2020) deverá responder aos desafios futuros que o ambiente e o uso dos seus recursos, o território em termos de desenvolvimento equilibrado, e a alimentação saberão colocar a escolha do home.

(10) Todavia «nei diversi PSR regionali, almeno dal punto di vista culturale ed "evocativo", il riferimento al paesaggio rurale è piuttosto frequente e tale da rivelare una crescita di consapevolezza sul tema, che si spera possa essere sviluppato in termini più concreti nelle programmazioni future» (Brunori, Fastelli, Rovai, 2013, p. 148).

\section{REFERÊNCIAS}

AGNOLETTI, Mauro (a cura di). Paesaggi rurali storici. Per un Catalogo Nazionale, Roma-Bari, Laterza, 2011.

AGNOLETTI, Mauro. Caratteristiche e stato di conservazione del paesaggio storico. In: AGNOLETTI (2011), pp. 5-103.

BIASUTTI, Renato. Il paesaggio terrestre, Torino, UTET, 1947.

BLASI, Giuseppe. Politiche, strumenti ed incentivi pubblici per la promozione dell'agricoltura multifunzionale. In FANFANI David (a cura di), Pianificare tra città e campagna. Scenari, attori e progetti di nuova ruralità per il territorio di Prato, Firenze, Firenze University, Press, 2009, pp. 67-72.

BRUNORI, Gianluca; FASTELLI, Laura; ROVAI, Massimo. Politiche di sviluppo rurale e paesaggio. In: POLI, Daniela (2013), pp. 129-160.

DEMATTEIS, Giuseppe. I piani paesistici: uno stimolo per ripensare il paesaggio geografico, In: «Rivista Geografica Italiana», XCVI, 1, 1989, pp. 446-451.

DI MARIO, Maurizio; PASCALE, Alfonso. Le campagne urbane e le nuove forme dell'abitare. In: GIARÈ, Francesca (a cura di), Mondi agricoli e rurali. Proposte di riflessione sui cambiamenti sociali e culturali, INEA, 2009, pp. 23-55 <http://www.inea.it/ap/bollettini/docs/mondiagricoli.pdf>.

DONADIEU, Pierre. Campagne urbane, una nuova proposta di paesaggio della città, Roma, Donzelli, 2004.

FACCIOLI, Marina. Processi territoriali e nuove filiere urbane, Milano, Angeli, 2009.

FARINELLI, Franco. L'arguzia del paesaggio. In: FARINELLI, I segni del mondo. Immagine cartografica e discorso geografico in età moderna, Firenze, La Nuova Italia, 1992, pp. 201-210.

GAMBINO Roberto. Il paesaggio tra conservazione e innovazione. In: DE ROSSI Antonio et alii (a cura di), Linee nel paesaggio. Esplorazioni nei territori della trasformazione, Torino, UTET, 1999, pp. 29-30. 
GOVERNA, Francesca; MEMOLI, Maurizio. Geografie dell'urbano. Spazi, politiche, pratiche della città, Roma, Carocci, 2011.

KRUSE, Alexandra, Agricultural landscape classification as tool for implementing the European Landscape Convention in research and planning results from the Eucalandproject. In: Living landscape. The European Landscape Convention in research perspective, Firenze, UNISCAPE, vol. 1, 2010.

LANZANI, Arturo. A piedi, immaginando un paesaggio per la geografia e l'urbanistica. In: Le frontiere della geografia, Torino, UTET, 2009, pp. 245-274.

MAGNAGHI Alberto. Il progetto locale. Verso la coscienze di luogo, Torino, Bollati Boringhieri, 2010.

MAGNAGHI Alberto. Il ruolo dei paesaggi rurali storici nella pianificazione territoriale. In AGNOLETTI (2011), pp. 111-120.

MAGNAGHI, Alberto; FANFANI David (a cura di). Patto città campagna. Un progetto di bioregione urbana per la Toscana centrale, Firenze, Alinea, 2010.

PETRILlO, Pier Luigi; DI BELlA, Ottavio; DI PALO, Nicola. La Convenzione UNESCO per il Patrimonio Mondiale e la valorizzazione dei paesaggi rurali. In: GOLINELLI, Gaetano M. (a cura di), Patrimonio culturale e creazione di valore, Padova, CEDAM, 2012, pp. 187- 246.

Piano Strategico Nazionale per lo Sviluppo rurale 2007-13, Ministero delle Politiche agricole, alimentari e forestali, Commissione europea, 2010 <http://www.reterurale.it/downloads/cd/PSN/Psn_21_06_2010.pdf >.

POLI, Daniela (a cura di).Agricoltura paesaggistica. Visioni, metodi, esperienze, Firenze, Firenze University Press, 2013.

POLLICE, Fabio (a cura di). Rapporto annuale della Società Geografica Italiana 2012. I nuovi spazi dell'agricoltura, Roma, Società Geografica Italiana, 2012.

QUAINI, Massimo (a cura di), Rapporto annuale della Società Geografica Italiana 2009. I paesaggi italiani. Fra nostalgia e trasformazione, Roma, Società Geografica Italiana, 2009.

RAFFESTIN, Claude. Dalla nostalgia del territorio al desiderio di paesaggio. Elementi per una teoria del paesaggio, Firenze, Alinea, 2005.

Regolamento (CE) n. 1698/2005 sul sostegno allo sviluppo rurale da pare del Fondo europeo agricolo per lo sviluppo rurale (FEASR). In: «Gazzetta ufficiale dell'Unione europea», L 277/1, 20 settembre 2005 <http://eurlex.europa.eu/LexUriServ/LexUriServ.do?uri=OJ:L:2005:277:0001:0040:IT:PDF>.

SALVATORI, Franco. Ilconsumo di suolo in Italia, un fenomeno di bulimia. In: «Ecoscienza», 4, 2011, pp. 20-21. 
SERENI, Emilio. Storia del paesaggio agrario italiano, Editori Laterza, Roma-Bari, 2010 .

SPAGNOLI, Luisa. Rappresentare e "agire" il paesaggio tra sostenibilità e nuove progettualità. Un itinerario geografico, Cagliari, Consiglio Nazionale delle Ricerche, 2012.

TEMPESTA, Tiziano. Paesaggio ed economia. In: AGNOLETTI (2011), pp. 133-146.

UNESCO, Convention concerning the protection of the world cultural and natural heritage.Paris, 16 november 1972 <http://whc.unesco.org/archive/conventionen.pdf $>$.

UNESCO, Operational Guidelines for the Implementation of the World Heritage Convention, 1992 <http://whc.unesco.org/archive/opguide92.pdf>.

VALLEGA,Adalberto. Paesaggio come prassi e rappresentazione. In: GHERSI, Adriana (a cura di), Politiche europee per il paesaggio: proposte operative, Roma, Gangemi, 2007, pp. 41-52.

VALLEGA, Adalberto. Indicatori per il paesaggio, Milano, Franco Angeli, 2008.

ZERBI, Maria Chiara. Paesaggi della Geografia, Giappichelli, Torino, 1993.

ZERBI, Maria Chiara. Il paesaggio rurale: un approccio patrimoniale, Torino, Giappichelli, 2007.

Artigo recebido para publicação em maio de 2014.

Artigo aceito para publicação em junho de 2014. 